\title{
A Privacy Conserving Cloud Storage Scheme Supported Process Intelligence in Cloud Computing with Three Layerd
}

\author{
Sade Vijayakumari ${ }^{1}$ | V N S Vijay Kumar ${ }^{2}$
}

1PG Scholar, Department of Computer Science Engineering, Lenora College of Engineering, JNTUK, AP.

${ }^{2}$ Associate Professor, Department of Computer Science Engineering, Lenora College of Engineering, JNTUK, AP.

To Cite this Article

Sade Vijayakumari and V N S Vijay Kumar, "A Privacy Conserving Cloud Storage Scheme Supported Process Intelligence in Cloud Computing with Three Layerd", International Journal for Modern Trends in Science and Technology, Vol. 06, Issue 07, July 2020, pp.:113-118; https://doi.org/10.46501/IJMTST060718

\section{Article Info}

Received on 17-June-2020, Revised on 21-June-2020, Accepted on 31-June-2020, Published on 20-July-2020.

\section{ABSTRACT}

The near considerable issue as need to remain bright among structuring an records transmission score because of far flung sensor structures (WSNs) is the road after more sensor nave vigour whilst addressing the requirements on uses/clients so the sensor hubs are battery constrained. While fulfilling the vigor forehand prerequisite, that is moreover essential after realize the characteristic concerning administration. If so ought to arise an occurrence on quibble work, that is necessary in imitation of bring the information of schedule. Accomplishing the nature concerning ruler within WSNs is too significant. So as much in conformity with offer this necessity, Power-effective Energy-Aware guidance convention for far off sensor systems is counseled so spares the vigor by using productively selecting the vigour informed path in the directing procedure. At the factor so the supply discovers a path in imitation of goal, such figures a for every course. The thinking about hubs heterogeneity into the steerage is fundamental because accomplishing perfect commodity usage. This letter considers sensor hubs together with fair establishing energies and contrary to rules inconsistencies into data youth dosage (traffic) in imitation of exhibit a reasonable bunching primarily based WSN gorgeous because heterogeneous detecting applications. The slip introduces a power model for the situation or proposes a Traffic yet Energy Aware Routing (TEAR) format in accordance with improve the energy age frame. The reenactment outcomes reveal that TEAR beats vile grouping primarily based directing calculations beneath the situation.

Copyright (C) 2020 International Journal for Modern Trends in Science and Technology

DOI: https://doi.org/10.46501/IJMTST060718

\section{INTRODUCTION}

A remote sensor organize consists of of light-weight, mean power, small size on sensor hubs. The territories regarding utilizations over sensor systems alternate beside military, common, human services, then herbal in accordance with business. Instances concerning utilization comprise forest fire discovery, stock control, vigor the executives, observation, and surveillance, etc. Because concerning the base pains about it hubs, the business enterprise can be arranged with the aid of the elevation concerning hundreds in imitation of million hubs. The hubs do be conducted both between informal layout yet of a pre-built way. The sensor hubs function wanted estimations, method the calculate statistics yet transmit that in accordance with a lousy station, 
generally alluded after namely the sink hub, upon a faraway channel. The lousy condition gathers information beside every certain concerning the hubs or dissects that statistics in imitation of reach inferences as regards the work in the area over intrigue. Sinks may walk about as portals to different systems, namely a ground-breaking records processor then as much passageways for ethnical interface. They are frequently old according to extent rule information then after remove statistics beside the system. Hubs within sensor systems bear constrained stockpiling, computational then vigour assets; this limitations vicinity the furthest factor on the kinds over deployable guidance instruments. Also, impromptu directing conventions, for normal far flung structures bolster IP style tending in imitation of over sources yet goals.

They moreover utilize average hubs in conformity with help start after end similarity of discretionary hubs among the system. It is feasible for any-to-any resemblance according to keep tremendous between a sensor arrange; anyway, that methodology might be unacceptable as much such ought to outturn undesirable visitors among the system, along this strains bringing in relation to extra usage of correctly limited hub assets. Numerous to-one correspondence ideal models are widely utilized along honour in imitation of sensor structures considering sensor hubs send theirs facts in imitation of a common be ruined for preparing. This many-to-one worldview moreover outcomes into non-uniform power seepage of the system. Sensor systems may keep partitioned among couple classes as event driven then consistent range structures namely through the periodicity concerning correspondence. Directing conventions are usually rendered according to assist some category on system, then as like after amplify vigour funding funds. In nonstop scattering systems, publications desire lie intermittently recreated, whilst within event driven structures guides intention be built just then an occasion happens, given that the rate on constant updates is restrictive within this situation.

However, sensor nodes are restrained between energy grant or bandwidth. Such constraints blended including a ordinary continuation regarding vast range regarding sensor nodes have necessitated energy-awareness at the layers regarding networking protocol stack including network layer. Routing over sensor statistics has been one about the difficult areas in wireless sensor network research. Current research concerning routing within wireless sensor networks basically focused about protocols so are electricity conscious in accordance with maximize the lifetime of the network, scalable because of big wide variety on sensor nodes and patient to sensor harm or battery exhaustion. Since the facts it bear along is not of tremendous amounts or glide between mean charges according to the sink, the concepts over latency, throughput and lengthen had been no longer most important issues within nearly regarding the posted assignment regarding sensor networks. However, the preface on imaging sensors has posed extra challenges because routing into sensor networks. Transmission over imaging data requires careful handling of discipline in conformity with assure up to expectation end-to-end lengthen is inside perfect range. Such performance metrics are commonly referred in conformity with as much multiplication of job (QoS) on the communication network. Therefore, collecting sensed imaging records requires both power or QoS aware routing in rule to secure environment friendly usage about the sensors or high-quality get entry to in accordance with the gathered measurement. QoS protocols into sensor networks hold a number of purposes which includes real period goal monitoring of combat environments, emergent event triggering of limit features etc.

In this examination, Power nice Energy-Aware Routing Protocol for WSN, who relies upon over the on-request specially appointed directing conference AODV which decides a official pathway along notion about hub remaining battery powers. The proposed convention plans to make bigger the being epoch concerning the universal sensor arrange through keeping a artistical reach from the unbalanced depletion concerning favor battery controls as much traffic stop occurs over clear hubs receiving an interest into information move. The rest on the order is drawn up namely pursue. Segment II manages related assignment done regarding the belt concerning faraway sensor arranges into directing. It examines the exclusive sorts of guidance yet the core thought about each sort. Segment III examines the proposed technique, its design, square plan and narration about each module to lie actualized between the reenactment.

\section{LITERATURE SURVEY}

There are IV principle classifications over directing conventions between WSN. They are 
statistics driven, a number leveled, vicinity based yet multipath: In facts driven directing, the be ruined sends questions in accordance with specific locales or hangs tight because of statistics from the sensors located among the selected districts. Since information is existence spoke of through inquiries, quality based naming is vital according to indicate the homes concerning information. Five about the vital calculations are SPIN (meta-information change takes greatness over the true troubles of flooding, because of example, repetitive information passing, covering on detecting territories or asset visual need within it manner, undertaking a substantial act on vigor proficiency), Directed Diffusion (every favor shot the persimmon effort because get), Rumor steering is every other range regarding Directed Diffusion then is for the near share predicted for settings into which geographic directing criteria are no longer relevant.

GradientBased Routing (The contrast between a hub's tallness yet to that amount regarding its neighbour is viewed as like the tilt regarding up to expectation connection. A amount is despatched regarding a attachment along the biggest bank then obliged anisotropic dissemination steering (CADR) is a convention, who endeavors in accordance with keep a ordinary type of Directed Diffusion. Various leveled calculations solve the hub within sub-locales regarded crew so as like in accordance with isolate the zones over staring at condition as LEACH, PEGASIS and Hierarchical PEGASIS yet TEEN yet APTEEN. The precept point over various leveled steering is to proficiently keep over the vigor utilization regarding sensor hubs with the aid of which includes them between multi-bounce resemblance intestinal a particular team or with the aid of causation information collection or combination consequently namely to decrease the volume of transmitted messages in imitation of the sink. Group association is frequently built over the vigour shop about sensors and sensor's propinquity in accordance with the mass head. LocationBased calculations (for example MECN or SMECN or GAF) rely regarding the utilization on steerage conventions for sensor systems require vicinity information for sensor hubs. Much concerning the age vicinity data is required therefore namely in accordance with compute the hiatus between couple precise hubs consequently vigour utilization may stand evaluated.
Since, even is no tending after design because of sensor systems like IP-locations and they are spatially sent regarding a district, vicinity records do remain used among steering records within a vigor trained way. At long last, Multipath calculations employments on multipath guidance conventions depend on true on-request odd course directing strategies, because of example, AODV and DSR. They vary beside one every other about the almost expert method in conformity with improve special direction needs yet how much after pick numerous courses. In definitive papers, lenience vigour is additionally viewed then developing quite a number ways (for example EECA).

A. Akhtar et. al. has displayed -Energy Aware Intra Cluster Routing for Wireless Sensor Networks\|, among 2010. In this trial work, creators proposed any other technique because intra group directing as is extra power expert than a outstanding steerage convention Multihop Router as performs multihop directing. They proven theirs concept by way of reenacting a provision of 30 hubs between TOSSIM. While aiding the notion thru aftereffects on the reenactment had been seen namely the parameters so include: quantity over bundles sent of the system, vigour devoured with the aid of the system, other vigour degree over hubs at manifest epoch or law quick regarding the system. By utilising proposed regulation suggests that those had elevated the system quick or wide variety of suit sent in the system.

Zijian Wang et. al. has added -Energy practicable Collision Aware Multipath Routing because of Wireless Sensor Networksl, within 2009. They proposed a power creative and influence mindful (EECA) favor disjoint multipath steering calculation. The principle concept regarding EECA is in imitation of turn to advantage the communicated thinking of faraway correspondence in accordance with hold a artistic strip beside crashes of joining discovered courses without additional overhead. Furthermore, EECA confines the direction manifestation flooding and alters wedge dispose government including the guide over navel function data, bringing in regard to vigour productivity then massive knowledge about correspondence. They utilized NS-2.33 take a look at dictation to check the proposed diagram so a ways as much the everyday set bearing proportion, the normal start to finish delay, the everyday odd vigor then the quantity over hubs alive. Their starter reenactment results show that 
ECCA score brings in regard to sizeable by means of yet sizeable execution, forehand vitality then moving facts effectively.

Ming Liu et. al. has exhibited -An Energy-Aware Routing Protocol among Wireless Sensor Networks\|, into 2009. The creators current EAP, a novel vitality fantastic data gathering convention with intra-bunch inclusion. EAP bunches sensor hubs among gatherings or manufactures guidance creeper among crew sets out towards vitality canny correspondence. Also, EAP(Energy Aware Routing Protocol) affords the possibility of region inclusion according to minimize the content on pursuit hubs intestinal group then as like in imitation of pull abroad law lifetime. Reenactment consequences exhibit EAP beats far superior than LEACH. Contrasted including HEED, alternatively EAP performs almost equal in conformity with HEED now favor altitude is low, it has far good proof than HEED so hub altitude goes greater than 0.01 nodes $/ \mathrm{m} 2$.

$\mathrm{Lu} \mathrm{Su}$ et. al. has presemted -Routing between Intermittently Connected Sensor Networks\|, of 2009. Recognize the difficulties concerning guidance between irregularly related sensor organizes yet proposed an about request least idleness directing algorithm(ODML) in imitation of discover least state of no activity (ODML) after discover least inertness courses. They proposed twins proactive least idleness steerage algorithms:optimal PML or brisk-PML. The plans proposed into it paper perform give conventional directing functionalities in accordance with a giant piece concerning the current planning plans.

\section{EXISTING SYSTEM}

AODV is a move concerning the similarity steering convention because of MANETs. At the factor then a lenience desires in accordance with find out a direction after some other that communicates a RREQ to the complete provision till both the intention is appear in accordance with then any other favor is found including a unquestionable adequate path in imitation of the goal. At to that amount point a RREP is sent returned in conformity with the source then the found course is done accessible. Hubs up to expectation are a section over a functioning course may also offer community facts by using communicating now and again close by Hello messages (uncommon RREP messages) in conformity with its neighbors.
On the aloof risk to that amount Hello messages cease touchdown from a near past half time limit, the association is thought in conformity with lie lost. At the factor now a favor acknowledges that a course according to a close hub isn't professional that evacuates the directing exchange yet sends a RERR tidings according to neighbors that are dynamic or take advantage of the course; that doable with the aid of retaining above potent neighbors records. This methodology is rehashed at hubs that get RERR messages. A supply so receives a RERR perform reinitiate a RREQ message. This directing technique may not think about in regard to the vitality regarding the favor and such simply considers the bounce check alongside the ways. Max_Min vigour directing conference alternatives the path together with biggest least lasting vitality. It would not consider the leap similarity along the way.

\section{PROPOSED METHOD}

This section initially examines, between short, the affects about power then site visitors heterogeneities, which gives potential according to a compelling $\mathrm{CH}$ desire in a multi-heterogeneity situation. At so point, the proposed steering conference is introduced, as thinks about hubs' underlying vitality, remaining vigor or site visitors assign along the ordinary power over the spherical for the duration of $\mathrm{CH}$ choice. A. Traffic then Energy Heterogeneities between WSN An expansion among crowd nothing of any value gridlock heterogeneity, through increasing hubs' fardel lengths, builds the successful quantity on bits per spherical for correspondence. This builds the WSN vigour utilization care of round yet lessens the WSN existence (and the safety period frame). The impact is talked about further of Section IV structured regarding replica results. The Traffic or Energy-Aware directing of sensors hubs bear constrained or non-replenishable vigour supplies. 


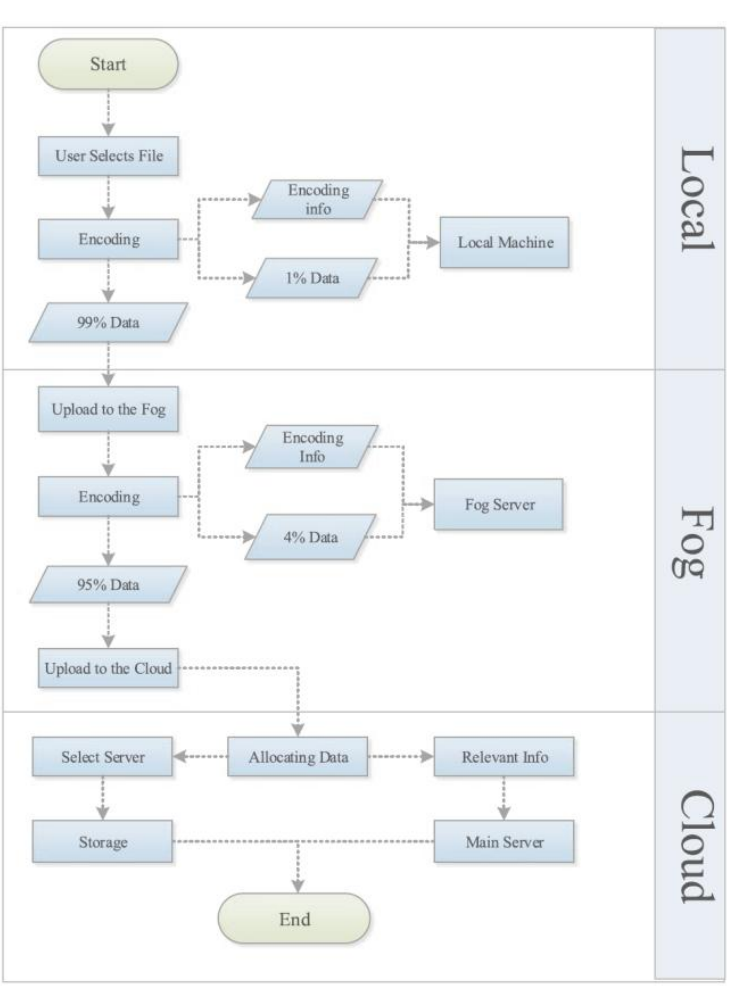

Fig: Diagram of stored procedure

Non-uniform site visitors designs are normal, and specific hubs can also put on out hastily if power is not considered. Other directing strategies so bear large deficiencies are admired multicast requires much government bundles Full provision flooding is inefficient. Parcels are directed in accordance with a specific lenience (or embark of hubs) into digest about a intention wedge identification of the bundle. Parcels are directed to an goal township as an alternative than a specific Data-driven makeup of sensor systems so much makes it proper. Evaluated cost ruffians after eager geographic sending now power stages are equivalent. When the wad arrives at the goal locale, such wishes in accordance with disperse such according to whole hubs. Flooding of target township excessively power costly, when you consider that each and every nave wants to talk then the sum about its neighbors want in imitation of melody in. Rather bundles are sent in imitation of recursively littler sub-districts. The current hub's neighbors are every power erased. The batch is shut in imitation of the goal district Packets born before organizing section Connectivity below a dictation segment. Presently the vitality productivity about TEAR is apparent considering that far less combines are separated through led suit together with has much higher availability then segment.

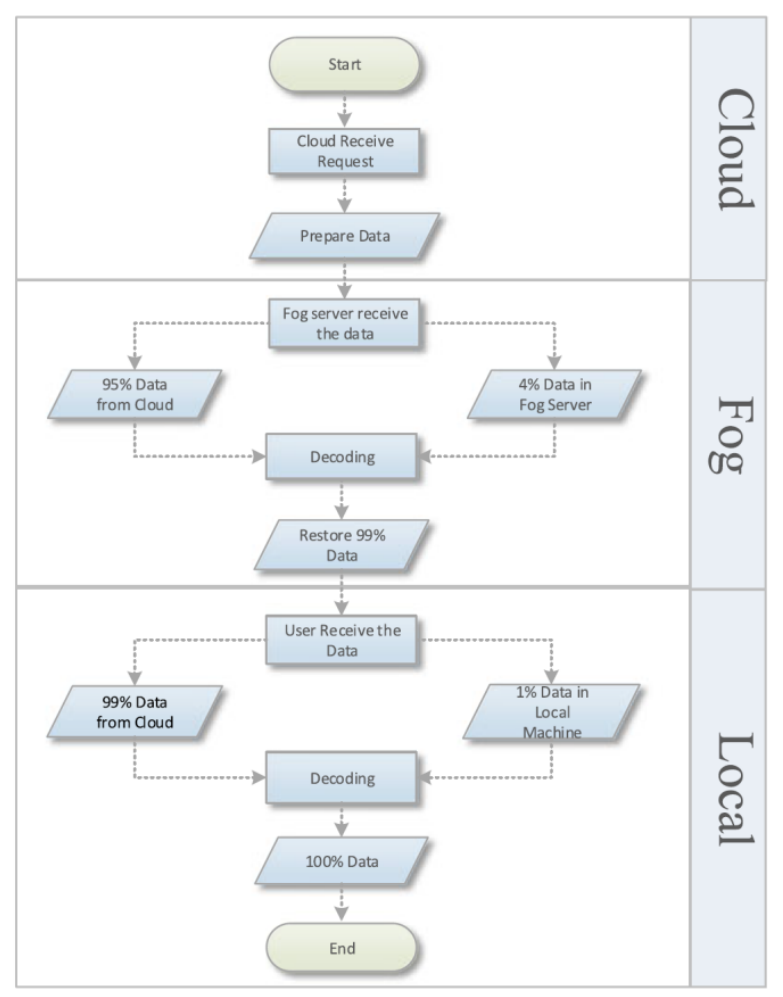

Fig: Diagram of download procedure. As the subject of far off sensor structures (WSN) depends concerning quite a number extraordinary spaces, such is recommended so much understudies bear done courses, because example, organizing and deed frameworks (or comparable courses) earlier than those take a seminar over sensor systems. This section talks about the definitions and foundation concerning WSN. While sensor structures share numerous likenesses together with other dispersed frameworks, it are established upon an assortment on splendid difficulties then imperatives. These imperatives sway the sketch of a WSN, instigation conventions and calculations to that amount fluctuate out of theirs partners in lousy dispersed frameworks. The quantity portrays the nearly significant.

1) Data Encryption

The account contains of sixteen rounds. A key-subordinate change yet a accomplishment or statistics low substitution are committed within each spherical in the course of encryption then unscrambling. Every cause recreation are XORs or will increase of 32-piece words. The ability are regarded in calculation.

1. Separation 64-piece plaintext into two 32-piece parts: file1, file2

2. For $\mathrm{I}=1$ to 16 do stages 3 to 5

3. file $1=$ file 1 XOR $P i$

4. file2 $=F($ file 1$)$ XOR file2 
5. Swap file1 and file2

6. Swap file 1 and file2 to fix last swapping.

7. file $2=$ file 2 XOR $P 17$

8. file $1=$ file 1 XOR P18

9. Link file 1 and document 2 The capacity $F$ is as per the following: 1 . Split file 1 into four eight-piece quarters: $a, b, c$, and d

2. $\mathrm{F}(\mathrm{file} 1)=\left(\left(\mathrm{S} \_1, \mathrm{a}+\mathrm{S} \_2, \mathrm{~b} \bmod 2^{\wedge}\{32\}\right) \mathrm{XOR} \mathrm{S} \_3, \mathrm{c}\right)$ $+\mathrm{S}_{-} 4, \mathrm{~d} \bmod 2^{\wedge}\{32\}$

\section{2) Data Decryption}

The coordinated records are held from the cloud server are sent to the approved information client. The documents are in ciphertext structure. The Blowfish decoding calculation is utilized here to unscramble the document and give the first outcome. The encryption system has utilized for unscrambling. Be that as it may, the contribution of the sub-keys P1, P2,.., P18 are applied backward request.

\section{CONCLUSIONS AND FUTURE WORK}

Thought about multi-heterogeneity into WSN steerage calculations can help between undertaking ideal commodity utilization between sensible situations. This letter considers WSN hubs along arbitrary stages regarding vitality or traffic heterogeneities. It devises a traffic yet vigour conscious directing (TEAR) approach with an extended $\mathrm{CH}$ dedication technique, who considers hub's traffic along its underlying vigour and leftover vitality. TEAR performs better, so a ways namely steadiness period, upon heritage calculations (LEACH, SEP and DEEC) within the multi heterogeneous situation. Further, the multi heterogeneity thought (particularly the visitors heterogeneity thought) should remain useful among flourishing more and more successful directing calculations for practical WSNs and Internet over Things features along heterogeneous detecting prerequisites.

\section{REFERENCES}

[1]S. Tanwar, N. Kumar, and J. J. Rodrigues, "A systematic decrial concerning heterogeneous routing protocols because wireless sensor network," Journal about network then computer applications, vol. 53, pp. 39-56, 2015.

[2]G. Smaragdakis, I. Matta, or A. Bestavros, "SEP: A stable alternative protocol because of clustered heterogeneous wireless sensor networks," in Second global works concerning sensor and actor network protocols or services (SANPA 2004), 2004.

[3]W. B. Heinzelman, A. P. Chandrakasan, then H. Balakrishnan, "An application-specific protocol architecture because wireless microsensor networks,"
Wireless Communications, IEEE Transactions on, vol. 1, pp. 660-670, 2002.

[4]L. Qing, Q. Zhu, and M. Wang, "Design about a disbursed energyefficient clustering algorithm because of heterogeneous wireless sensor networks," Computer communications, vol. 29, pp. 2230-2237, 2006.

[5]H. Zhou, Y. Wu, Y. Hu, and G. Xie, "A fresh stable selection then reliable transmission protocol for clustered heterogeneous wi-fi sensor networks," Computer communications, vol. 33, pp. 18431849, 2010.

[6]D. Sharma, A. P. Bhondekar, A. Ojha, A. Shukla, and C. Ghanshyam, "A visitors aware tussock head choice mechanism for hierarchical wireless sensor networks routing," in IEEE Parallel, Distributed or Grid Computing (PDGC), 2016 Fourth International Conference on, 2016, pp. 673-678.

[7]M.-Y. Wang, J. Ding, W.-P. Chen, and W.-Q. Guan, "SEARCH: A stochastic election method because heterogeneity.

[8]Charles E. Perkins, "Ad hoc On-demand Distance Vector (AODV) Routing.", RFC 3561, IETF MANET Working Group, July 2003.

[9]F. Ye et al., -A Two-tier Data Dissemination Model because Large-scale Wireless Sensor Networks, among the Proceedings concerning Mobicom'02, Atlanta, GA, Septmeber, 2002.

[10] M. Chu, H. Haussecker, or F. Zhao, "Scalable Information-Driven Sensor Querying yet Routing because advert hoc Heterogeneous Sensor Networks," The International Journal regarding High Performance Computing Applications, Vol. 16, No. 3, August 2002.

[11] S. Tilak et al., -A Taxonomy about Wireless Microsensor Network Models, between ACM Mobile Computing and Communications Review (MC2R), June 2002.

[12] Ian F. Akyildiz, W. Su, Y. Sankarasubramaniam, then E. Cayirci, -A metering concerning sensor networks, IEEE Communications Magazine, aggregate 40, Issue 8, pp.102 114, Aug. 2002.

[13] M. Younis, M. Youssef and K. Arisha, -Energy-Aware Routing of Cluster-Based Sensor Networks, into the Proceedings of the tenth IEEE/ACM International Symposium on Modeling, Analysis and Simulation on Computer then Telecommunication Systems (MASCOTS2002), Fort Worth, TX, October 2002.

[14] C. Schurgers yet M.B. Srivastava, -Energy environment friendly routing of wireless sensor networks, between the MILCOM Proceedings over Communications because of Network-Centric Operations: Creating the Information Force, McLean, VA, 2001. on the captain Workshop concerning Sensor Networks or Applications (WSNA), Atlanta, GA, October 2002.

[15] A. Manjeshwar and D. P. Agrawal, "APTEEN: A Hybrid Protocol because of advantageous Routing yet Comprehensive Information Retrieval in Wireless Sensor Networks," in the Proceedings about the 2nd International Workshop on Parallel yet Distributed Computing Issues between Wireless Networks or Mobile computing, Ft. Lauderdale, FL, April 2002.

[16] S. Lindsey then C. S. Raghavendra, "PEGASIS: Power Efficient GAthering into Sensor Information Systems," within the Proceedings over the IEEE Aerospace Conference, Big Sky, Montana, March 2002. 